\title{
ON A MATCHING METHOD FOR A LINEAR ORDINARY DIFFERENTIAL EQUATION CONTAINING A PARAMETER, I
}

\author{
By Toshiniko Nishimoto
}

\section{§1. Introduction.}

Let a linear ordinary differential equation of the form

$$
\varepsilon^{h} \frac{d y}{d x}=A(x, \varepsilon) y,
$$

be given, where $h$ is a positive integer, $x$ is a complex independent variable, $\varepsilon$ is a complex parameter, and $A(x, \varepsilon)$ is an $n$-by- $n$ matrix of the form

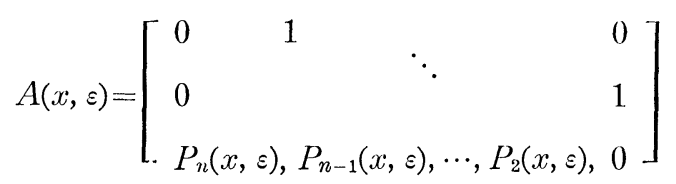

The functions $P_{k}(x, s)(k=2, \cdots, n)$ are holomorphic in the domain of the $x, \varepsilon$ space defined by the inequalities,

$$
|x| \leqq x_{0}<1, \quad 0<|\varepsilon| \leqq \varepsilon_{0}, \quad|\arg \varepsilon| \leqq \delta_{0}
$$

and each of the functions $P_{k}(x, \varepsilon)$ has a uniformly asymptotic expansion in powers of $\varepsilon$ such that

$$
P_{k}(x, \varepsilon) \simeq \sum_{\nu=0}^{\infty} P_{k \nu}(x) \varepsilon^{\nu}
$$

as $\varepsilon$ tends to zero in the domain (1.3) with holomorphic coefficients $P_{k \nu}(x)$ :

$$
P_{k \nu}(x)=\sum_{\mu=m_{k \nu}}^{\infty} x^{\mu} P_{k \nu \mu}, P_{k \nu m_{k \nu}} \neq 0,
$$

where $P_{k \nu \mu}$ are constant and $m_{k \nu}$ are non negative integers. Suppose that $m_{k 0} \geqq 1$ for $k=2, \cdots, n$, then the equation (1.1) has a turning point at the origin. We consider here the asymptotic behavior of solutions of equation (1.1) in the neighborhood of the turning point as $\varepsilon$ tends to zero. When $A(x, \varepsilon)$ is a 2 -by-2 matrix, it was treated in this author's previous paper [2], and in this paper we shall generalize that results to the equations of higher order.

Assume that

Received June 18. 1965. 


$$
m_{n 0}=q \geqq 1 \text {, }
$$

$$
\begin{aligned}
& \frac{m_{k \nu}}{k} \geqq \frac{q}{n}-\frac{1}{a} \cdot \frac{\nu}{k}, \quad \text { for } \quad \begin{array}{l}
\nu=0,1, \cdots, \\
k=2, \cdots, n,
\end{array} \\
& \frac{m_{k \nu}}{k}>\frac{q}{n}-\frac{1}{a} \cdot \frac{\nu}{k}, \quad \text { for } \quad \begin{aligned}
\nu & =1,2, \cdots, \\
k & =2, \cdots, n,
\end{aligned}
\end{aligned}
$$

where

$$
a=\frac{n h}{n+q} .
$$

Under these hypotheses with further restrictions described in later, we can obtain the two types of asymptotic representations of the fundamental solutions of the equation (1.1) whose domains of validity are overlapped with each other for arbitrarily small $\varepsilon$ in some neighborhoods of the turning point. In the next paper, we remove the assumption (1.8).

In Sections 2 and 3, we calculate the two types of formal solutions, in Sections 4 and 5 it will be proved that there exist fundamental solutions whose asymptotic expansions coincide with the formal solutions in some neighborhoods of the turning point, and in Section 6 we conclude that in several subdomains which overlap the full neighborhood of the turning point, we can calculate the asymptotic expansions of the fundamental solutions.

\section{§2. Formal solutions for $x \neq 0$.}

The linear transformation introduced originally by Iwano and Sibuya [1],

$$
y=\Omega(x) u,
$$

where

$$
\Omega(t)=\left[\begin{array}{cccc}
1 & & & 0 \\
& t^{q^{\prime n}} & & \\
& & \dot{t}^{(n-1) q^{\prime} n} &
\end{array}\right]
$$

changes the equation (1.1) into

$$
\begin{aligned}
& \varepsilon^{h} x^{-q^{\prime n}} \frac{d u}{d x}=\left\{\left[\begin{array}{ccrr}
0 & 1 & \ddots & 0 \\
0 & & & 1 \\
\left(x^{q / n}\right)^{-n} P_{n}(x, \varepsilon), & \left(x^{q / n}\right)^{-(n-1)} P_{n-1}(x, \varepsilon), \cdots, & 0
\end{array}\right]\right.
\end{aligned}
$$

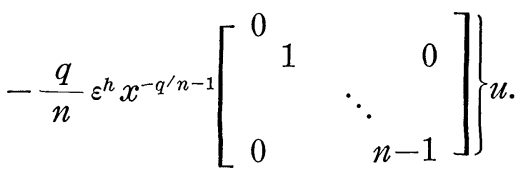

By (1.4) and (1.5) we have 
(2. 4)

$$
\left(x^{q / n}\right)^{-k} P_{k}(x, \varepsilon) \simeq \sum_{\nu=0}^{\infty} \sum_{\mu=m_{k \nu}}^{\infty} P_{k \nu \mu} x^{\mu-q k / n} \cdot \varepsilon^{\nu}
$$

$$
\simeq \sum_{\nu=0}^{\infty} \sum_{\mu=m_{k}}^{\infty} P_{k_{\nu \mu}}\left[x^{-1 / a} \varepsilon\right]^{\nu} x^{\nu / a+\mu-k q / n}
$$

where $\nu / a+\mu-k q / n=\nu / a+k(\mu / k-q / n) \geqq 0$ from (1.7), and $\nu / a+\mu-k q / n=0$ if and only if $\nu=0, \mu / k=q / n$ from (1.8).

Then the equation (2.3) takes the form

$$
\left[x^{-1 / a} \varepsilon\right]^{h} x \frac{d u}{d x}=B(x, \varepsilon) u,
$$

where

$$
B(x, \varepsilon) \simeq \sum_{\nu=0}^{\infty} B_{\nu}(x)\left[x^{-1 / a} \varepsilon\right]^{\nu}
$$

with

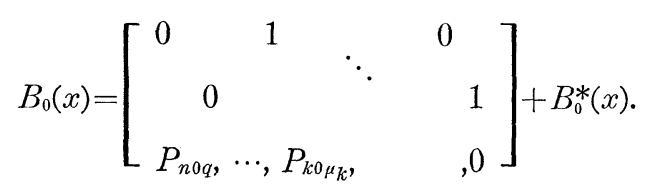

We notice here that for nonzero elements $P_{k 0 \mu_{k}}$, we have $\mu_{k}=k q / n$, and $B_{0}^{*}(x)$ and $B_{\nu}(x)(\nu \geqq 1)$ are holomorphic matrices functions of $x^{1 / a b}$, where $b$ is a positive integer for which $a b \cdot k q / n$ is equal to integer for $k=2, \cdots, n$. (2.6) means that for every $m$

$$
B(x, \varepsilon)-\sum_{\nu=0}^{m} B_{\nu}(x)\left[x^{-1 / a} \varepsilon\right]^{\nu}=E_{m}(x, \varepsilon)\left[x^{-1 / a} \varepsilon\right]^{m+1},
$$

where $E_{m}(x, \varepsilon)$ is bounded in the domain (1.3).

Here we put

$$
x=\tau^{a b},
$$

then (2.6) becomes

$$
\left[\tau^{-b} \varepsilon\right]^{h} \tau \frac{d u}{d \tau}=C(\tau, \varepsilon) u
$$

where $C(\tau, \varepsilon)$ is holomorphic in $\tau$ and $\varepsilon$ for

$$
|\tau| \leqq \tau_{0}, \quad 0<|\varepsilon| \leqq \varepsilon_{0}, \quad|\arg \varepsilon| \leqq \delta_{0},
$$

and has an asymptotic expansion when $\varepsilon$ tends to zero:

$$
C(\tau, \varepsilon) \simeq \sum_{\nu=0}^{\infty} C_{\nu}(\tau)\left[\tau^{-b} \varepsilon\right]^{\nu}
$$

The matrices functions $C_{\nu}(\tau)$ are holomorphic for $|\tau| \leqq \tau_{0}$, and 


$$
C_{0}(\tau)=\alpha b\left[\begin{array}{ccrc}
0 & 1 & & 0 \\
& 0 & \ddots & 1 \\
P_{n o q}, \cdots, P_{k 0 \mu_{k}}, \cdots, & 0
\end{array}\right]+C_{0}^{*}(\tau) .
$$

Let $\lambda_{1}, \cdots, \lambda_{n}$ be the characteristic roots of the matrix $(a b)^{-1} C_{0}(0)$ and assume that

$$
\lambda_{2} \neq \lambda_{j}, \quad i \neq j, \quad i, j=1, \cdots, n .
$$

In this case we can prove the following lemma.

Lemma 2. 1. Under the condition (2.12), there exists a linear transformation

$$
u=P(\tau, \varepsilon) z
$$

which changes the equation (2.8) inlo

$$
\left[\tau^{-b} \varepsilon\right]^{h} \tau \frac{d z}{d t}=D(\tau, \varepsilon) z
$$

with the following properties:

a) $D(\tau, \varepsilon)$ is holomorphic in $\tau$ and $\varepsilon$ for

(2.15) $\quad|\tau| \leqq \tau_{0}, \quad|\arg \tau| \leqq \alpha_{0}, \quad 0<|\varepsilon| \leqq \varepsilon_{0} \quad|\arg \varepsilon| \leqq \delta_{0}, \quad 0<\left|\tau^{-b} \varepsilon\right| \leqq \mu_{0}$, for sufficiently small positive numbers $\tau_{0}$ and $\mu_{0}$, and arbilrary $\alpha_{0}$.

b) As $\left|\tau^{-b} \varepsilon\right|$ tends to zero, we have

$$
D(\tau, \varepsilon) \simeq \sum_{\nu=0}^{\infty} D_{\nu}(\tau)\left[\tau^{-b} \varepsilon\right]^{\nu}
$$

uniformly in (2.15).

c) The matrices $D_{\nu}(\tau)$ are diagonal and holomorphe for $|\tau| \leqq \tau_{0}$,

d)

$$
D_{0}(\tau)=\left[\begin{array}{ccc}
\lambda_{1}(\tau) & & 0 \\
& \ddots & \\
0 & & \lambda_{n}(\tau)
\end{array}\right], \lambda_{i}(\tau)=\lambda_{i}+o(\tau) .
$$

e) The matrix $P(\tau, \varepsilon)$ is holomorphic in $\tau$ and $\varepsilon$ for (2.15) and

$$
P(\tau, \varepsilon) \simeq \sum_{\nu=0}^{\infty} P_{\nu}(\tau)\left[\tau^{-b} \varepsilon\right]^{\nu},
$$

where $P_{0}(\tau)$ is nonsingular and $P_{\nu}(\tau)$ holomorphic for $|\tau| \leqq \tau_{0}$.

The proof is almost same as [2] and then is omitted here. (see also Wasow [4]).

Since all the matrices $D_{\nu}(\tau)$ of $(2.16)$ are diagonal, we can calculate a formal series solution of the differential equation (2.14) and get a following theorem.

THEOREM 2.1. The differential equation (2.8) possesses a formal matrix solution of the form 


$$
u \sim \sum_{\nu=0}^{\infty} \varepsilon^{\nu} u_{\nu}(\tau) \exp \left[\sum_{\nu=0}^{h} \varepsilon^{\nu-h} F_{\nu}(\tau)\right]
$$

with the properties;

$$
u_{\nu}(\tau)=\tau^{-b \nu} \hat{u}_{\nu}(\tau)
$$

where $\hat{u}_{\nu}(\tau)$ are polynomials of degree $\nu$ at most in $\log \tau$ whose coefficients are holomorphic in $|\tau| \leqq \tau_{0}$, and bounded in the domain (2.15).

$$
\begin{gathered}
F_{\nu}(\tau)=\tau^{-b(\nu-h)} \hat{F}_{\nu}(\tau) \quad \nu \leqq h-1 \\
F_{h}(\tau)=f_{r} \log \tau+\hat{F}_{h}(\tau)
\end{gathered}
$$

where $\hat{F}_{\nu}(\tau)(\nu=0,1, \cdots, h)$ are holomorphic in $|\tau| \leqq \tau_{0}$, and $f_{h}$ is a constant matrix.

The proof of this theorem is similar to that of [2], Theorem 1, and then is omitted here.

\section{$\S 3$. Formal solutions in the neighborhoods of $x=0$.}

We transform the equation (1.1) by stretching and shearing transformation of the form, (see Iwano and Sibuya [1])

$$
\begin{aligned}
& x=\varepsilon^{a} S \\
& y=\Omega\left(\varepsilon^{a}\right) v,
\end{aligned}
$$

where $\Omega\left(\varepsilon^{a}\right)$ is defined by (2.2) with $\varepsilon^{a}$ instead of $t$, and then becomes

$$
\frac{d v}{d s}=\left[\begin{array}{ccccc}
0 & 1 & \ddots & & \\
0 & & & & 1 \\
\varepsilon^{-n \gamma} p_{n}\left(\varepsilon^{a} s, \varepsilon\right), & \cdots, & \varepsilon^{-2 r} p_{2}\left(\varepsilon^{a} s, \varepsilon\right), & 0
\end{array}\right] v .
$$

where $\gamma=q \alpha / n$.

From (1.4) and (1.5). we have

$$
\varepsilon^{-k_{r}} p_{k}\left(\varepsilon^{a} S, \varepsilon\right) \simeq \sum_{\nu=0}^{\infty} \sum_{\mu=m_{k \nu}}^{\infty} p_{k \nu \mu} S^{\mu} \varepsilon^{\nu+a_{i t}-k r}
$$

and remark here that from (1.7) and (1.8),

$$
\nu+a \mu-k \gamma=a k\left\{\frac{1}{a} \frac{\nu}{k}+\frac{\mu}{k}-\frac{q}{n}\right\} \geqq 0,
$$

$$
\nu+a \mu-k \gamma=0 \quad \text { if and only if } \nu=0 \text { and } \mu=\mu_{k}=\frac{k q}{n} \text {. }
$$

We put

$$
\varepsilon=\rho^{n+q}
$$

with $\rho>0$ for $\arg \varepsilon=0$.

Thus the equation (3.3) can be written 


$$
\frac{d v}{d s}=H(s, \rho) v
$$

where $H(s, \rho)$ is holomorphic in $s$ and $\rho$ for (1. 3), and has asymptotic expansion in (1.3) and $|s| \leqq s_{0}$ for arbitrary $s_{0}$ such that

$$
H(s, \rho)=\sum_{\nu=0}^{\infty} H_{\nu}(s) \rho^{\nu}
$$

where $H_{\nu}(s)$ are holomorphic matrices functions of the forms

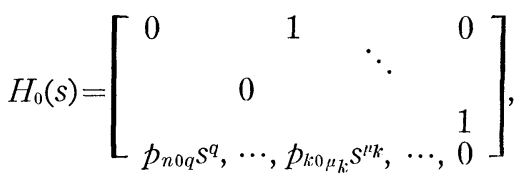

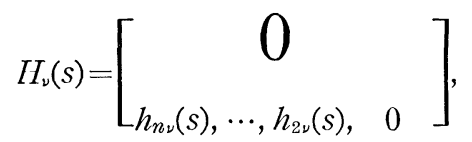

Now we consider the asymptotic properties of $I I(s, \rho)$ in the domain (1.3) and $|s| \geqq s_{0}$.

Let $H_{\nu}^{* *}(s)$ be

$$
H_{\nu}^{* *}(s)=\Omega(s)^{-1} H_{\nu}(s) \Omega(s), \quad(\nu \geqq 1) .
$$

Then from (3.11) and (2.2)

$$
H_{\nu}^{* *}(s)=\left[\begin{array}{cc}
0 \\
s^{-(n-1) q / n} p_{n \nu}(s), \cdots, s^{-q^{\prime n}} p_{2 \nu}(s), & 0
\end{array}\right],
$$

but from (3.4) and (3.7), the function $h_{k \nu}(s)$ is a polynomial of $s$ of degree at most $k q / n+\nu / n h$, and then $H_{\nu}^{* *}(s)$ can be written

$$
H_{\nu}^{* *}(s)=s^{\nu / n h+q / n} H_{\nu}^{*}(s)
$$

where $H_{\nu}^{*}(s)$ is bounded at $s=\infty$. Thus $H_{\nu}(s)$ can be written

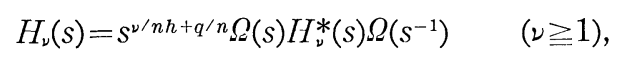

where $H_{\nu}^{*}(s)$ is bounded at $s=\infty$.

LEEMA 3. 1. For every $m \geqq 0$, there exists a matrix function $E_{m+1}(s, \rho)$, bounded in the domain (1.3) and $|s| \geqq s_{0}$ such that

$$
H(s, \rho)-\sum_{\nu=0}^{m} H_{\nu}(s) \rho^{\nu}=s^{q / n} \Omega(s) E_{m+1}(s, \rho) \Omega\left(s^{-1}\right)\left[s^{1 / n h} \rho\right]^{m+1} .
$$

Proof. To prove this lemma, it is sufficient for us to state that for every $m \geqq 0$, there exists a bounded function $e_{m+1}(s, \rho)$ in the domain (1.3) and $|s| \geqq s_{0}$ such that 


$$
\varepsilon^{-k r} p_{k}\left(\varepsilon^{a} S, \varepsilon\right)-\sum_{\nu=0}^{m} h_{k \nu}(S) \rho^{\nu}=S^{k q / n+(m+1) / n h} e_{m+1}(s, \rho) \cdot \rho^{m+1} .
$$

But this is easily derived by considering the order of magnitude of the remainder terms, and the details are rendered to the previous paper [2].

Let

$$
v \sim \sum_{\nu=0}^{\infty} v_{\nu}(s) \rho^{\nu}
$$

be a formal solution of (3.3). Then $v_{\nu}(s)$ must satisfy the following equations:

$$
\begin{aligned}
& \frac{d v_{0}}{d s}=H_{0}(s) v_{0}, \\
& \frac{d v_{\nu}}{d s}=H_{0}(s) v_{\nu}+\sum_{\mu=1}^{\nu} H_{\mu}(s) v_{\nu-\mu} .
\end{aligned}
$$

The asymptotic solution of (3.16) can be obtained from the recent results of Okubo [3]. Now we translate the Okubo's main results into the terminology of the differential equation (3.16).

If we transform the equation (3.16) by the equations

$$
\begin{aligned}
\xi & =\frac{n}{n+q} s^{(n+q) / n}, \\
v_{0} & =\Omega(s) \mathfrak{w},
\end{aligned}
$$

we have

$$
\xi \frac{d w}{d \xi}=(A+\xi B) w
$$

where

$$
\begin{aligned}
& A=-\frac{q}{n+q}\left[\begin{array}{rrrr}
0 & & & 0 \\
& 1 & & \\
& & \ddots & \\
& 0 & & n-1
\end{array}\right], \\
& B=\left(\begin{array}{cccc}
0 & 1 & & \\
& & \ddots & \\
& 0 & & \\
p_{n o q}, \cdots, & p_{k 0 \mu_{k}}, \cdots, & 0
\end{array}\right) .
\end{aligned}
$$

Here we suppose the following assumptions on the matrices $A$ and $B$.

a) Let $\lambda_{1}, \cdots, \lambda_{n}$ be the characteristic roots of $B$, and choose $\arg \lambda_{2}$ in such a way that $-\pi<\arg \lambda_{\imath} \leqq \pi, i=1, \cdots, n$. Then, we assume in addition to (2.12),

$$
\begin{array}{cl}
\lambda_{3} \neq 0, & j=1, \cdots, n, \\
\left|\arg \lambda_{i}-\arg \lambda_{j}\right|>0, & i \neq j, \quad i, j=1, \cdots, n .
\end{array}
$$


b) Let $P$ be a constant matrix such that $P^{-1} B P=\wedge$ is diagonal, and let $I I$ be a diagonal matrix whose diagonal elements $\pi_{\imath}$ are the diagonal elements of $P^{-1} A P$. In this case, we assume

$$
-\frac{k q}{n+q}-\pi_{\imath} \neq-1,-2, \cdots, i=1, \cdots, n, k=0,1, \cdots, n-1,
$$

c)

$$
\frac{q(k-l)}{n+q}=0, \pm 1, \pm 2, \cdots, \quad k \neq l, k, l=0,1, \cdots, n-1 .
$$

Under these conditions, the differential equation (3.18) has a fundamental matrix solution in the neighborhood of $\xi=0$ of the form

$$
w_{0}=\left[\sum_{\nu=0}^{\infty} w_{0 \nu} \xi^{\nu}\right] \xi^{A}, \quad \operatorname{det} w_{00} \neq 0
$$

with constant matrices $w_{0 \nu}$, and in the neighborhood of $\xi=\infty$, there exist several asymptotic matrices solutions in the sectorial domains of the form

$$
W_{\infty} \simeq\left[\sum_{\nu=0}^{\infty} w_{\infty \nu} \xi^{-\nu}\right] \xi^{\pi} e^{\wedge \xi}, \quad \operatorname{det} w_{\infty 0} \neq 0
$$

with constant matrices $w_{\infty \nu}$.

The connection formulas of the solutions (3.19) and (3.20) can be obtained in several sectorial neighborhoods of $\xi=\infty$ which overlap the full neighborhood of $\xi=\infty$. One of such sectorial neighborhoods is given by

$$
\Sigma(\theta)=\bigcap_{i=1}^{n}\left\{\left|\arg \lambda_{i} \xi\right| \leqq \frac{3}{2} \pi-\beta\right\},
$$

where $\beta$ is arbitrarily small positive number. The sector $\Sigma(\theta)$ contains a sector whose central angle is at least $\pi$. Let $\Sigma$ be a sector contained in $\Sigma(\theta)$ such that

$$
\Sigma:-\alpha \leqq \arg \xi \leqq-\alpha+\pi, \quad\left(0 \leqq \alpha<\frac{\pi}{2}\right),
$$

where the number $\alpha$ is specified in later.

Thus we have an asymptotic solution of the equation (3.16) of the form

$$
v_{0}=\Omega(s) w_{0}(s) s^{R} \exp [Q(s)],
$$

where

$$
R=\frac{n+q}{n}\left[\begin{array}{ccc}
\pi_{1} & & \\
& \ddots & \\
0 & & \pi_{n}
\end{array}\right]=\left[\begin{array}{lll}
\gamma_{1} & & \\
& & 0 \\
& \ddots & \\
0 & & \gamma_{n}
\end{array}\right]
$$




$$
Q(s)=\frac{n}{n+q} s^{(n+q) / n}\left[\begin{array}{ccc}
\lambda_{1} & & 0 \\
& \ddots & \\
0 & & \lambda_{n}
\end{array}\right]=\left[\begin{array}{cc}
q_{1}(s) & 0 \\
& \ddots \\
0 & q_{n}(s)
\end{array}\right],
$$

$$
w_{0}(s) \simeq \sum_{\nu=0}^{\infty} w_{0 \nu} s^{-(n+q) \nu / n}, \quad \operatorname{det} w_{00} \neq 0,
$$

with constant matrices $w_{0 v}$, and this is valid in the domain $S\left(s_{0}\right)$ :

$$
|s| \geqq s_{0},
$$

$$
\frac{-\alpha n}{n+q} \leqq \arg s \leqq \frac{n(\pi-\alpha)}{n+q}
$$

Now we must solve the differential equation (3.17) which is of the form

$$
\frac{d t}{d s}=H_{0}(s) t+F(s)
$$

with entire coefficients. The integral

$$
t(s)=\int_{\Gamma(s)} v_{0}(s) v_{0}{ }^{-1}(\sigma) F(\sigma) d \sigma
$$

is a solution of (3.28) if $\Gamma(s)$ designates a set of paths $\gamma_{j k}(s)$ in $\sigma$-plane ending at $\sigma=s$ for every scalar integral contained in (3.29). The paths $\gamma_{j k}(s)$ will be given in later.

Define $\hat{t}(s), \hat{v}_{0}(s)$ and $\hat{F}(s)$ by the relations

$$
\begin{gathered}
t(s)=\Omega(s) \hat{\boldsymbol{t}}(s) s^{R} \exp [Q(s)], \\
v_{0}(s)=\Omega(s) \hat{v}_{0}(s) s^{R} \exp [Q(s)], \\
F(s)=\Omega(s) \hat{F}(s) s^{R} \exp [Q(s)] .
\end{gathered}
$$

Then, (3. 29) becomes

$$
\hat{t}(s)=\hat{v}_{0}(s) \int_{\Gamma(s)}\{\exp [Q(s)-Q(\sigma)]\}(s / \sigma)^{R} \hat{v}_{0}(\sigma)^{-1}
$$

$$
\cdot \hat{F}(\sigma)(\sigma / s)^{R} \exp [Q(\sigma)-Q(s)] d \sigma .
$$

Now assume that for some positive number $b$,

$$
\hat{F}(s) s^{-b} \quad \text { is bounded in } S\left(s_{0}\right) \text {, }
$$

then

$$
\hat{\boldsymbol{v}}_{0}(s)^{-1} \hat{\boldsymbol{F}}(s) s^{-b} \quad \text { is bounded in } S\left(s_{0}\right) .
$$

To simplify the notations, we define $q_{j k}(s)$ and $\beta_{j k}$ by

$$
q_{j k}(s)=q_{j}(s)-q_{k}(s)=\frac{n}{n+q} s^{(n+q) / n}\left(\lambda_{j}-\lambda_{k}\right)=\beta_{j k} s^{(n+q) / n} .
$$


From (3.33), (3.35) and (3.36), every element of the matrix in the integrand of (3.33) has a form

$$
p_{j k}(\sigma) \sigma^{b} \cdot(s / \sigma)^{r_{j}-r_{k}} \exp \left[\beta_{j k}\left(s^{(n+q) / n}-\sigma^{(n+q) / n}\right)\right],
$$

where $p_{j k}(\sigma)$ is bounded in $S\left(s_{0}\right)$. We consider this integral in the $\xi$-plane and introduce the auxiliary variables

$$
\zeta=\frac{n}{n+q} \sigma^{(n+q) / n}, \quad \xi=\frac{n}{n+q} s^{(n+q) / n} .
$$

The sector $S$ defined by (3.27) in the $\sigma$-plane corresponds to a half plane $\Sigma$ in the $\zeta$-plane. Now we choose the angle $\alpha$ in (3.20) in such a way that every line,

$$
\operatorname{Re}\left[\left(\lambda_{j}-\lambda_{k}\right) \zeta\right]=0 \quad j \neq k, \quad j, k=1, \cdots, n
$$

does not coincide with boundary lines of $\Sigma$, and clearly this is always possible. Then every line (3.39) has one half line inside $\Sigma$. Hence for every pair $(j, k), j \neq k$, we can draw a ray $l_{j k}$ through the origin into the interior of $\Sigma$ such that $\operatorname{Re}\left[\left(\lambda_{j}-\lambda_{k}\right) \zeta\right]$ increases monotonically to $\infty$ along $l_{j k}(j \neq k)$ as $|\zeta|$ increases to $\infty$. Denote by $\lambda_{j k}(\xi)(j \neq k)$ the straight half line in $\Sigma$ which is parallel to $l_{j k}$ and has one end point at a point $\xi$ of $\Sigma$. Then $\operatorname{Re}\left[\left(\lambda_{j}-\lambda_{k}\right) \zeta\right]$ also increases monotonically to $\infty$ along $\lambda_{j k}(\xi)$. We define $\gamma_{j k}(s), j \neq k$, as the curve in the sector $S$ of the $\sigma$-plane whose image under (3.38) is $\lambda_{j k}(\xi)$. Then $\operatorname{Re} q_{j k}(\sigma)$ grows monotonically to $\infty$ as $\sigma$ increases to $\infty$ along $\gamma_{j k}(s)$.

In order to make sure that all points of $\gamma_{j k}(s)$ lie in the domain $S\left(s_{0}\right)$ of the $\sigma$-plane, we must limit $s$ to a domain $S\left(s_{1}\right)$, where $s_{1}$ is sufficiently large. For the paths $\gamma_{j j}(s)$, it suffices to take them as segments from some points $s_{2}$ in $S\left(s_{1}\right)$ to $s \in S\left(s_{1}\right)$, where $s_{2}$ is so large that these segments lie in $S\left(s_{0}\right)$ for all $s \in S\left(s_{1}\right)$.

LEMMA 3.2. If the differential equation (3.28) satisfies the condition (3.34), then it possesses a solution of the form

$$
t(s)=s^{b+1} \Omega(s) t^{*}(s) s^{R} \exp [Q(s)]
$$

where $t^{*}(s)$ is bounded as $s \rightarrow \infty$ in $S$.

Proof. If (3.37) is integrated along $\gamma_{j k}(s)$, it becomes in terms of $\zeta$ and $\xi$,

$$
\begin{gathered}
\frac{n}{n+q} \xi^{\pi j-\pi_{k}} \int_{\lambda_{k j}(\xi)}\left\{\exp \left(\lambda_{j}-\lambda_{k}\right)(\xi-\zeta)\right\} \tilde{p}_{j k}(\sigma) \zeta^{\left(\pi_{k}-r_{j}\right)} \zeta^{(n b-q) /(n+q)} d \zeta, \\
j, k=1,2, \cdots, n .
\end{gathered}
$$

If we express $\zeta$ on $\lambda_{j k}(\xi)$ in the form

$$
\zeta=\xi+\delta_{j k} r, \quad j, k=1,2, \cdots, n,
$$

where $\delta_{j k}$ is a constant of modulus 1 and $r$ is a real variable, then (3.41) becomes

$$
\begin{gathered}
\frac{n}{n+q} \xi^{(n b-q) /(n+q)} \int_{0}^{\infty}\left\{\exp -\left(\lambda_{j}-\lambda_{k}\right) \delta_{j k} r\right\} \tilde{p}_{j k}(\sigma)\left[1+\delta_{j k} \frac{r}{\xi}\right]^{\pi_{k}-\pi_{j}+(n b-p) /(n+q)} \delta_{j k} d r, \\
j, k=1,2, \cdots, n .
\end{gathered}
$$


For $j \neq k, \operatorname{Re}\left(\lambda_{j}-\lambda_{k}\right) \delta_{j k}>0$ on $\lambda_{j k}(\xi)$ and thus the integral in (3.42) is a uniformly bounded function of $\xi$ for $s \in S\left(s_{1}\right)$. Then (3.42) is of the order $O\left(s^{b-q / n}\right)$ as $s \rightarrow \infty$ in $S\left(s_{1}\right)$. For $j=k, \beta_{j k}=0$ in (3.37), and then integral of (3. 37) along $\gamma_{j j}(s)$ is $O\left(s^{b+1}\right)$. Thus Lemma 3.2 follows at once using the relation (3.30).

Lemma 3. 3. The differential equation (3.17) possesses a particular solution of the form

$$
v_{\nu}(s)=s^{e \nu} \Omega(s) w_{\nu}(s) s^{R} \exp [Q(s)],
$$

where $w_{\nu}(s)$ is bounded as $s \rightarrow \infty$ in the sector $S$, and

$$
e=\frac{1}{n h}+\frac{q}{n}+1 \text {. }
$$

Proof. We prove this by induction. For $\nu=0$, the equation $(3.17)$ becomes the equation (3.16) and the statement in Lemma 3. 3 is satisfied from (3.22). Assume it to be true for $\nu<m$. The $\mu$-th term of the summation in (3.17) has a form

$$
H_{\mu}(s) v_{m-\mu}(s)=s^{f(m, \mu)} \Omega(s) H_{\mu}{ }^{*}(s) w_{m-\mu}(s) s^{R} \exp [Q(s)],
$$

where $H_{\mu}(s)$ is the expression defined by (3.12), and

$$
f(m, \mu)=\frac{\mu}{n h}+\frac{q}{n}+(m-\mu) e .
$$

The exponent $f(m, \mu)$ is the largest for $\mu=1$, and then for $\nu=m$ we can apply Lemma 3.2 to the equation (3. 17) with

$$
b=f(m, 1) \text {. }
$$

Thus we get the following theorem.

Theorem 3.1. We assume on the matrices $A$ and $B$ in (3. 18) the conditions a), b), c) and (2.12), and let $k(s)$ be defined by

$$
k(s)=\left\{\begin{array}{lll}
0 & \text { if } & |s| \leqq s_{0}, \\
1 & \text { if } & |s|>s_{0} .
\end{array}\right.
$$

Then the differential equation (3.8) has a formal matrix solution $v$ of the form

$$
v \sim \Omega\left(s^{k(s)}\right)\left\{\sum_{\nu=0}^{\infty} w_{\nu}(s)\left[s^{k(s) e} \cdot \rho\right]^{\nu}\right\} s^{k(s) R} \exp [Q(s)] .
$$

where $w_{\nu}(s)$ are bounded in the domain (1.3) and $|s| \leqq s_{0}$ with $k(s)=0$, and in the domain (1.3), $|s|>s_{0}$ with $k(s)=1$.

REMARK 1. Among the assumptions on the matrices $A$ and $B$ stated in above Theorem, the essential conditions are the condition (2.12) and the condition (b). In fact the connection formulas of the solutions (3.19) with (3.20) can be obtained without the condition a) and c) by a little modification. (Okubo [3].)

But we assume to hold all the conditions stated in Theorem 3.1 for simplicity's sake. 
REMARK 2. Let $C$ be the connection matrix between the solution of the equation (3.16) in the neighborhood of $s=0$ and the asymptotic solution (3.22) in the sector (3. 27), and let $v_{\nu}{ }^{(0)}, v_{\nu}{ }^{(\infty)}$ be the solutions of (3.17) in the neighborhood of $s=0$, $s=\infty$ respectively. Then, we can construct the solution $v_{\nu}{ }^{(0)}$ such that

$$
v_{\nu}{ }^{(0)} \cdot C=v_{\nu}{ }^{(\infty)}, \quad \nu=0,1, \cdots .
$$

To prove this statement, we consider the equation (3.28) with

$$
\begin{array}{ll}
\frac{d t^{(0)}}{d s\rceil}=H_{0}(s) t^{(0)}+F(s) & |s| \leqq s_{0} \\
\frac{d t^{(\infty)}}{d s}=H_{0}(s) t^{(\infty)}+F(s) C & |s|>s_{0} .
\end{array}
$$

If $t^{0}(s)$ is a solution of (3. 49), then $t^{(0)}(s) C$ is a solution of (3.50). Hence if we determine the solution $t^{(0)}(s)$ by the condition

$$
t^{(0)}\left(s_{1}\right) C=t^{(\infty)}\left(s_{1}\right)
$$

for some point $s_{1}$, we can conclude (3.48). And this is always possible for

$$
t^{(0)}(\$)=\int v_{0}(s) v_{0}(\sigma)^{-1} F(\sigma) d \sigma+v_{0}(s) \cdot P
$$

and $t^{(\infty)}(s)$ which is constructed in Lemma 3.2 by taking constant matrix $P$ appropriately. Therefore two formal solutions of (3. 8)

$$
\begin{gathered}
v^{(0)} \sim\left\{\sum_{\nu=0}^{\infty} w_{\nu}{ }^{(0)}(s) \rho^{\nu}\right\} \exp [Q(s)], \\
v^{(\infty)} \sim \Omega(s)\left\{\sum_{\nu=0}^{\infty} w_{\nu}^{(\infty)}(s)\left[s^{e} \rho\right]^{\nu}\right\} s^{R} \exp [Q(s)]
\end{gathered}
$$

are connected formally by the relation

$$
v^{(\infty)} \sim v^{(0)} \cdot C
$$

\section{§4. Existence Theorem (1).}

Here we prove the following theorem.

THeOREm 4.1. Let $T$ be any sector of $\tau$-plane with vertex at the origin and central angle less than $\pi / b h$ ( $b$ is the number introduced in (2.7), and different from that of (3. 34)), and let

$$
u \sim \sum_{\nu=0}^{\infty} \varepsilon^{\nu} u_{\nu}(\tau) \exp \left[\sum_{\nu=0}^{h} \varepsilon^{\nu-h} F_{\nu}(\tau)\right]
$$

be a formal solution of (2.8) whose existence was shown in Theorem 2.1. Then, there exists an actual solution of (2.8) of the form 


$$
u(\tau, \varepsilon)=\hat{u}(\tau, \varepsilon) \exp \left[\sum_{\nu=0}^{h} \varepsilon^{\nu-h} F_{\nu}(\tau)\right]
$$

and for every positive integer $m$, there exists a domain $D_{1}$ of $\varepsilon$, $\tau$-plane defined by

$$
\tau \in T, \quad 0<|\varepsilon| \leqq \varepsilon_{1}, \quad|\arg \varepsilon| \leqq \delta_{1}, \quad c_{1}|\varepsilon|^{1 / b} \leqq|\tau| \leqq c_{2},
$$

$\left(\varepsilon_{1}, \delta_{1}, c_{1}\right.$ and $c_{2}$ are certain constants depending on $m$ but independent of $\left.\varepsilon\right)$ in which it holds

$$
\hat{u}(\tau, \varepsilon)-\sum_{\nu=0}^{m} u_{\nu}(\tau) \varepsilon^{\beta}=E_{m}(\tau, \varepsilon)\left[\tau^{-b} \varepsilon\right]^{m+1}
$$

where $E_{m}(\tau, \varepsilon)$ is a matrix function bounded in the domain (4.1).

Proof. We divide this proof into several stages. At first we analyze the equation (2.14).

Let the matrices functions $D_{m}(\tau, \varepsilon)$ and $z^{(m)}(\tau, \varepsilon)$ be

$$
\begin{gathered}
D_{m}(\tau, \varepsilon)=\sum_{\nu=0}^{m+h} D_{\nu}(\tau)\left[\tau^{-b} \varepsilon\right]^{\nu}, \\
z^{(m)}(\tau, \varepsilon)=\exp \left\{\sum_{\nu=0}^{m+h} \varepsilon^{\nu-h} F_{\nu}(\tau)\right\} .
\end{gathered}
$$

Then $z^{(m)}(\tau, \varepsilon)$ is a fundamental solution of an equation

$$
\left(\tau^{-b} \varepsilon\right)^{h} \tau \frac{d z}{d \tau}=D_{m}(\tau, \varepsilon) z
$$

By the transformation

$$
z=z^{(m)}+w^{(m)}
$$

the equation (2.14) becomes

$$
\left(\tau^{-h} \varepsilon\right)^{h} \tau \frac{d w^{(m)}}{d \tau}=D(\tau, \varepsilon) w^{(m)}+\left[D(\tau, \varepsilon)-D_{m}(\tau, \varepsilon)\right] z^{(m)},
$$

and if we define $K(\tau, \varepsilon), D_{h}(\tau, \varepsilon), \hat{w}^{(m)}(\tau, \varepsilon)$ and $\hat{z}^{(m)}(\tau, \varepsilon)$ by

$$
\begin{aligned}
K(\tau, \varepsilon) & =\sum_{\nu=0}^{h} \varepsilon^{\nu} F_{\nu}(\tau), \\
D_{h}(\tau, \varepsilon) & =\sum_{\tau=0}^{h} D_{\nu}(\tau)\left[\tau^{-b} \varepsilon\right]^{\nu}, \\
\hat{w}^{(m)}(\tau, \varepsilon) & =w^{(m)}(\tau, \varepsilon) \exp \left[-\varepsilon^{-h} K(\tau, \varepsilon)\right], \\
\hat{z}^{(m)}(\tau, \varepsilon) & =z^{(m)}(\tau, \varepsilon) \exp \left[-\varepsilon^{-h} K(\tau, \varepsilon)\right],
\end{aligned}
$$

then (4.8) becomes

$$
\begin{aligned}
\left(\tau^{-b} \varepsilon\right)^{h} \tau \frac{d \hat{w}^{(m)}}{d \tau}= & D_{h}(\tau, \varepsilon) \hat{w}^{(m)}-\hat{w}^{(m)} D_{h}(\tau, \varepsilon)+\left[D(\tau, \varepsilon)-D_{h}(\tau, \varepsilon)\right] \hat{w}^{(m)} \\
& +\left[D(\tau, \varepsilon)-D_{m}(\tau, \varepsilon)\right] \hat{z}^{(m)} .
\end{aligned}
$$


Here we have

$$
\begin{aligned}
& D(\tau, \varepsilon)-D_{h}(\tau, \varepsilon)=\left(\tau^{-b} \varepsilon\right)^{h+1} E_{h}(\tau, \varepsilon), \\
& D(\tau, \varepsilon)-D_{m}(\tau, \varepsilon)=\left(\tau^{-b} \varepsilon\right)^{m+h+1} E_{m}(\tau, \varepsilon),
\end{aligned}
$$

where $E_{h}(\tau, \varepsilon)$ and $E_{m}(\tau, \varepsilon)$ are bounded in (2.15), and from (4. 5), (4.9) and (4.12), we can see

$$
\hat{z}^{(m)}=\exp \left[\sum_{\nu=h+1}^{m+h} \varepsilon^{\nu-h} F_{\nu}(\tau)\right]=\exp \left[\sum_{\nu=h+1}^{m+h}\left(\tau^{-b} \boldsymbol{\varepsilon}\right)^{\nu-h} \widetilde{F}_{\nu}(\tau)\right]
$$

with bounded matrices functions $\widetilde{F}_{\nu}(\tau)$, and then $\hat{z}_{m}$ is bounded in (2.15).

Now we write the differential equation (4.13) for each component of $\hat{w}^{(m)}(\tau, \varepsilon)=$ $\left(\hat{w}_{i j}^{(m)}(\tau, \varepsilon)\right)$, that is

$$
\left(\tau^{-b} \varepsilon\right)^{h} \tau \frac{d \hat{w}_{i j}^{(m)}}{d \tau}=\left(d_{i}-d_{j}\right) \hat{w}_{i j}^{(m)}+\left[\left(D-D_{h}\right) \hat{w}^{(m)}+\left(D-D_{m}\right) \hat{z}^{(m)}\right]_{\imath \jmath} .
$$

where $d_{\imath}$ are the $i$-th diagonal element of $D_{h}(\tau, \varepsilon)$, and this differential equation can be converted by the method of variation of constants into the following integral equation

$$
\begin{aligned}
\hat{w}_{i j}{ }^{(m)}(\tau, \varepsilon)= & \varepsilon^{-h} \int_{r_{i j}}\left\{\exp \varepsilon^{-h}\left[\mu_{i j}(\tau)-\mu_{i j}(\sigma)\right]\right\}\left\{\left[D(\sigma, \varepsilon)-D_{h}(\sigma, \varepsilon)\right] \hat{w}^{(m)}(\tau, \varepsilon)\right. \\
& \left.+\left[D(\sigma, \varepsilon)-D_{m}(\sigma, \varepsilon)\right] \hat{z}^{(m)}(\sigma, \varepsilon)\right\}_{{ }^{\prime} j} \sigma^{b h-1} d \sigma,
\end{aligned}
$$

where

$$
h_{i j}(\tau, \varepsilon)=K_{i}(\tau, \varepsilon)-K_{j}(\tau, \varepsilon)
$$

with diagonal elements $K_{i}(\tau, \varepsilon)$ of $K(\tau, \varepsilon)$, and $\gamma_{i}$ is an integral path which is chosen so that the exponential function in (4.17) remains bounded as $\varepsilon$ tends to zero.

Now we prove the existence of the solution of the equation $(4.17)$ by the fixed point theorem.

Let $F$ be the set of all matrices functions $W(\tau, \varepsilon)=\left(w_{\imath j}(\tau, \varepsilon)\right)$ whose components are holomorphic in $\tau$ and $\varepsilon$ for (4.2) and satisfy the inequality

$$
\| W(\tau, \varepsilon)|| \leqq M\left|\tau^{-b} \varepsilon\right|^{m+1},
$$

where

$$
\|W\|=\max _{1 \leqq i \leqq n}\left[\sum_{\jmath=1}^{n}\left|w_{\imath j}\right|\right],
$$

and the constant $M$ will be chosen appropriately. $F$ is closed, compact, and convex with respect to the topology of uniform convergence on each compact subset of the domain (4.2). The mapping $T(w)$ is defined by right hand term of (4.17). Thus it is sufficient for us to prove that the mapping $T$ is continuous and has a contracting property. The continuity follows at once if we choose the integral paths $\gamma_{i j}$ in such a way that the integral (4.17) converges uniformly for all matrices functions of $F$. 
From (4. 14), (4.15) and the boundedness of $\hat{z}_{m}(\tau, \varepsilon)$, there exist constant $M_{h}, M_{m}$ and $B_{m}$ and $B$ independent of $\varepsilon, \tau$ and $\tau^{-b} \varepsilon$ such that

$$
\begin{aligned}
& \left\|D(\tau, \varepsilon)-D_{h}(\tau, \varepsilon)\right\| \leqq M_{h}\left|\tau^{-b} \varepsilon\right|^{h+1}, \\
& \left\|D(\tau, \varepsilon)-D_{m}(\tau, \varepsilon)\right\| \leqq M_{m}\left|\tau^{-b} \varepsilon\right|^{m+h+1}, \\
& \left\|\hat{z}^{(m)}(\tau, \varepsilon)\right\| \leqq B,
\end{aligned}
$$

in the domain (2.15). Then we have

$$
\left|T\left(w_{\imath j}\right)\right| \leqq|\varepsilon|^{m+1} \int_{r_{\imath \jmath}}\left\{\exp \varepsilon^{-h}\left[\mu_{i j}(\tau)-\mu_{i j}(\sigma)\right]\right\}\left(M_{h} M\left|\sigma^{-b} \varepsilon\right|+M_{m} B\right)|\sigma|^{-b\left(m_{\top} 1\right)-1} d \sigma .
$$

Here we must take $\left|\tau^{-b} \varepsilon\right|$ sufficiently small, that is

$$
\left|\tau^{-b} \varepsilon\right| \leqq \mu_{1}
$$

and this is achieved by taking $c_{1}$ in (4.2) large enough.

Now we can choose the constants $\varepsilon_{1}, \delta_{1}, c_{1}$ and $c_{2}$ in (4.2) and the integral paths $\gamma_{\imath j}(\tau)(i, j=1, \cdots, n)$ in (4. 17) in such a way that the $\operatorname{Re}\left[\varepsilon^{-h} \mu_{i j}(\tau, \varepsilon)\right]$ is monotonic decreasing along $\gamma_{i j}(\tau)$ and a following inequality holds for $\tau$ in the domain (4.2)

$$
\int_{\gamma_{i j}(\tau)}|\sigma|^{-b(m+1)-1}|d \sigma| \leqq M_{1}|\tau|^{-b(m+1)}
$$

where $M_{1}$ is dependent on $m$ but not on $\varepsilon, \tau_{0}$ and $\mu_{1}$. These statements can be proved by the same methods in [2] and the proof is omitted. Hence we have from (4. 20),

$$
\left|T\left(w_{i j}\right)\right| \leqq\left[M_{h} M \mu_{1}+M_{m} B\right] M_{1}\left|\tau^{-b} \varepsilon\right|^{m+1},
$$

and if $\mu_{1}$ in (4.21) is taken so small and $M$ so large that

$$
n\left[M_{h} M \mu_{1}+M_{m} B\right] M_{1} \leqq M,
$$

so we can conclude that ||$T(w)|| \leqq M\left|\tau^{-b} \varepsilon\right|^{m+1}$ and also from the uniform convergence of the integral, $T(w)$ is holomorphic, that is

$$
T(F) \subset F \text {. }
$$

Thus we prove the existence of the solution of (4.17) and then (4.16) or (4.13) of the form

$$
\hat{w}^{(m)}(\tau, \varepsilon)=\left(\tau^{-b} \varepsilon\right)^{m+1} E(\tau, \varepsilon)
$$

where $E(\tau, \varepsilon)$ is bounded in (4.2).

Now we prove that the solution $\hat{w}^{(m)}(\tau, \varepsilon)$ of $(4.16)$ such that

$$
\hat{w}^{(m)}(\tau, \varepsilon)=0\left(\tau^{-b} \varepsilon\right)^{m+1}
$$

is unique. Assume the contrary, and let $\varphi=\left(\varphi_{i j}(\tau, \varepsilon)\right)$ be the difference of any two solutions. Then, evidently the integral equation

$$
\varphi_{i j}(\tau, \varepsilon)=\varepsilon^{-h} \int_{r_{i j}}\left\{\exp \varepsilon^{-h}\left[\mu_{i j}(\tau)-\mu_{i j}(\sigma)\right]\right\}\left[D(\sigma, \varepsilon)-D_{h}(\sigma, \varepsilon)\right] \varphi_{i j}(\sigma, \varepsilon) \sigma^{b h-1} d \sigma
$$


must hold. If we put

it follows that

$$
\max _{\substack{D_{1} \\ 1 \leqq j \leqq n}}\left|\varphi_{i j}(\tau, \varepsilon)\left(\tau^{-b} \varepsilon\right)^{-(m+1)}\right| \equiv K>0,
$$

$$
\left|\varphi_{i j}(\tau, \varepsilon)\right| \leqq K \cdot M_{h} \cdot M_{1}\left|\tau^{-b} \varepsilon\right| \cdot\left|\tau^{-b} \varepsilon\right|^{m+1} .
$$

Therefore if we choose $\mu_{1}$ in (4.21) so small that $M_{h} M_{1} \mu_{1}<1$, we obtain $K<K$ which is a contradiction. Hence $K \equiv 0$ and the unicity of the solution is established.

Next we consider the solution of the equation (2.14):

$$
z(\tau, \varepsilon, m)=z^{(m)}(\tau, \varepsilon)+w^{(m)}(\tau, \varepsilon) .
$$

Then, we can prove that the solution $z(\tau, \varepsilon, m)$ is independent of $m$. Let $m^{\prime}>m$, $D_{1}\left(m^{\prime}\right), D_{1}(m)$ be corresponding domains which are dependent on $\mu_{1}$ in (4.15) and hence on $m$, and

$$
\begin{aligned}
z(\tau, \varepsilon, m) & =\left[\hat{z}^{(m)}(\tau, \varepsilon)+\hat{w}^{(m)}(\tau, \varepsilon)\right] \exp \left[\varepsilon^{-h} K(\tau, \varepsilon)\right], \\
z\left(\tau, \varepsilon, m^{\prime}\right) & =\left[\hat{z}^{\left(m^{\prime}\right)}(\tau, \varepsilon)+\hat{w}^{\left(u^{\prime}\right)}(\tau, \varepsilon)\right] \exp \left[\varepsilon^{-h} K(\tau, \varepsilon)\right] .
\end{aligned}
$$

Clearly the function

$$
\hat{z}^{\left(m^{\prime}\right)}(\tau, \varepsilon)+\hat{w}^{\left(m^{\prime}\right)}(\tau, \varepsilon)-\hat{z}^{(m)}(\tau, \varepsilon)
$$

satisfies the differential equation (4.16), and in the domain $D_{1}(m) \cap D_{1}\left(m^{\prime}\right)$ and $\mu_{1}$ in (4. 21) sufficiently small it satisfies the inequality (4.18). It follows from the unicity established above that

$$
\hat{w}^{(m)}(\tau, \varepsilon)=\hat{z}^{\left(m^{\prime}\right)}(\tau, \varepsilon)+\hat{w}^{\left(m^{\prime}\right)}(\tau, \varepsilon)-\hat{z}^{(m)}(\tau, \varepsilon),
$$

and then

$$
z(\tau, \varepsilon, m)=z\left(\tau, \varepsilon, m^{\prime}\right) .
$$

Thus we have established Theorem 4.1 for the solutions of the differential equation (2.14), and concerning the solutions of the differential equation (2.8), the statements of Theorem 4.1 can be easily obtained with some modifications on each constants $\varepsilon_{1}, \delta_{1}, c_{1}$ and $c_{2}$ and on $E_{m}(\tau, \varepsilon)$, by the same methods in [2].

\section{$\S 5$. Existence Theorem (2).}

In this Section we prove the existence theorem corresponding to the formal solution (3.47) in Theorem 3.1.

THEOREM 5.1. Let

$$
\begin{aligned}
v & \sim \sum_{\nu=0}^{\infty} v_{\nu}(s) \rho^{\nu} \\
& \sim \Omega\left(s^{k(s)}\right)\left\{\sum_{\nu=0}^{\infty} w_{\nu}(s)\left(s^{k(s) e} \rho\right)^{\nu}\right\} s^{k(s) R} \exp [Q(s)]
\end{aligned}
$$


be a formal solution of the differential equalion (3.8) whose exislence was proved in Theorem 3.1. Then, for every positive inleger $m$, there exists a domain $D_{2}$ of the $s, \rho$-plane defined by

$$
s \in S, \quad 0<|\rho| \leqq \rho_{2}, \quad|\arg \rho| \leqq \delta_{2}, \quad\left|s^{c} \rho\right| \leqq c_{3},
$$

where $\rho_{2}, \delta_{2}$ and $c_{3}$ are some constants depending on $m$ and $e$ is a number defined by (3. 44), and there exists a fundamental matrix solution $v(s, \rho)$ of the equation (3. 8), which is related to the formal solution by the formula

$$
v(s, \rho)-\sum_{\nu=0}^{m} v(s) \rho^{\nu}=\Omega\left(s^{k(s)}\right) E_{m}(s, \rho)\left[s^{k(s) e} \rho\right]^{m+1} s^{k(s) R} \exp [Q(s)],
$$

where $E_{m}(s, \rho)$ is a matrix function bounded in the domain (5.1), and $k(s), \Omega(s), R$, $Q(s)$ are defined in (3.46), (2.2), (3.23), (3.24) respectively. The solution $v(s, \rho)$ is independent of $m$.

Proof. Let

$$
v^{(m)}(s, \rho)=\sum_{\nu=0}^{m} v_{\nu}(s) \rho^{\nu}
$$

be the finite sum of the series (3. 47). This satisfies at differential equation

$$
\frac{d v}{d s}=H_{m} v, \quad H_{m}=v^{(m)^{\prime}} \cdot\left(v^{m}\right)^{-1},
$$

where $v^{(m)^{\prime}}$ denotes the derivative of $v^{(m)}$ with respect to $s$.

Clearly $v_{0}(s)$ is a nonsingular matrix function and all $v_{\nu}(s)$ are entire matrices functions. Hence if $s_{0}>0$ is chosen arbitrarily, $v_{m}(s, \rho)^{-1}$ exists for

$$
|\rho| \leqq \rho_{1}, \quad|s| \leqq s_{0},
$$

where $\rho_{1}$ is sufficiently small positive number depending on $s_{0}$ and $m$. On the other hand, by Lemma 3. 3 we have

$$
v^{(m)}(s, \rho)=\Omega(s)\left[\sum_{\nu=0}^{m} w_{\nu}(s)\left(s^{e} \rho\right)^{\nu}\right] s^{R} \exp [Q(s)],
$$

where $w_{\nu}(s)$ are bounded for $|s|>s_{0}$ and $s \in S$, and $w_{0}(s)$ is nonsingular for $s \neq 0$ from (3. 22), and the nonsingularity of $v_{0}(s)$. Then it follows from $(5.6)$ that $v^{(m)}(s, \rho)^{-1}$ exists for $s \in S$ and

$$
\left|s^{e} \rho\right| \leqq \eta_{1}, \quad|s|>s_{0},
$$

where $\eta_{1}$ is sufficiently small positive number depending on $s_{0}$ and $m$.

Define a function $v^{(m)}(s, \rho)$ by the equation

$$
v^{(m)}(s, \rho)=\Omega\left(s^{k(s)}\right) \hat{v}^{(m)}(s, \rho) s^{k(s) R} \exp Q(s),
$$

where $k(s)$ is defined in (3.46). Then from the above discussions, the matrix function $\hat{\boldsymbol{v}}^{(m)}(s, \rho)$ is bounded and nonsingular if $s$ and $\rho$ satisfy the condition (5.5) or (5. 7).

If we put 


$$
v=v^{(m)}+z^{(m)},
$$

the equation (3.8) becomes

$$
\frac{d z^{(m)}}{d s}=H(s, \rho) z^{(m)}+H(s, \rho) v^{(m)}-v^{(m)} .
$$

Then any solution of the integral equation

$$
\begin{aligned}
z^{(m)}(s, \rho)= & \int_{\Gamma(s)} v_{0}(s, \rho) v_{0}(\sigma, \rho)^{-1}\left\{\left[I I(\sigma, \rho)-I I_{0}(\sigma, \rho)\right] z^{(m)}(\sigma, \rho)\right. \\
& \left.+\left[H(\sigma, \rho) v^{(m)}(\sigma, \rho)-v^{(m) '}(\sigma, \rho)\right]\right\} d \sigma
\end{aligned}
$$

satisfies the differential equation (5.10). Here $\Gamma(s)$ denotes a set of $n^{2}$ paths of integration in the $\sigma$-plane ending at $s$.

If $\hat{z}^{(m)}(s, \rho)$ is defined by

$$
z^{(m)}(s, \rho)=\Omega\left(s^{k(s)}\right) \hat{z}^{(m)}(s, \rho) s^{k(s) R} \exp [Q(s)],
$$

then (5.11) becomes

$$
\begin{aligned}
\hat{z}^{(m)}(s, \rho)=w_{0}(s) \int_{\Gamma(s)} s^{k(s) R}\{\exp [Q(s)-Q(\sigma)]\} \sigma^{-k(\sigma) R} w_{0}(\sigma)^{-1} \Omega\left(\sigma^{-k(\sigma)}\right) \\
\quad\left\{\left[H(\sigma, \rho)-H_{0}(\sigma, \rho)\right] \Omega\left(\sigma^{k(\sigma)}\right) \hat{z}^{(m)}(\sigma, \rho) \sigma^{k(\sigma) R} \exp [Q(\sigma)]\right. \\
\left.\quad+\left[H(\sigma, \rho) v^{(m)}(\sigma, \rho)-v^{(m)}(\sigma, \rho)^{\prime}\right]\right\} \exp [-Q(s)] s^{-k(s) R} d \sigma .
\end{aligned}
$$

Here we need an estimate of $H(\sigma, \rho) v^{(m)}(\sigma, \rho)-v^{(m)}(\sigma, \rho)^{\prime}$.

$$
\begin{aligned}
H I v^{(m)}-v^{(m)} & =\left\{\sum_{\nu=0}^{m} H_{\nu} \rho^{\nu}+R_{m}\right\} \sum_{\nu=0}^{m} v_{\nu} \rho^{\nu}-\sum_{\nu=0}^{m} v_{\nu}{ }^{\prime} \rho^{\nu} \\
& =\sum_{\nu=0}^{m} \rho^{\nu}\left\{\sum_{\mu=1}^{\nu} H_{\mu} v_{-\mu}-v_{\nu}{ }^{\prime}\right\}+\sum^{*} H_{\mu} v_{\nu-\mu} \rho^{\nu}+R_{m} v^{(m)},
\end{aligned}
$$

where the summation $\Sigma^{*}$ is for

$$
\mu \leqq m, \quad \nu-\mu \leqq m, \quad \nu>m, \quad \mu \geqq 1,
$$

and $R_{m}$ denotes the remainder terms in the series expansion of $H(s, \rho)$. Among the last three expressions in (5.14), the first term is zero, because $v_{\nu}$ is the solution of (3.17). From (3.12) and (3.43), the second term can be written

$$
\Omega\left(s^{k(s)}\right) \Sigma^{*} \rho^{\nu} S^{k(s) g(\nu, \mu)} H_{\mu}{ }^{*} w_{\nu-\mu} s^{k(s) R} \exp [Q(s)],
$$

where $g(\nu, \mu)=\mu / n h+q / n+(\nu-\mu) e$. The maximum number of $g(\nu, \mu)$ for each $\nu$ is attained by $\mu=1$, that is

$$
\frac{\mu}{2 h}+\frac{q}{n}+(\nu-\mu) e \leqq \nu e-1,
$$

and so the summation (5.12) can be written

$$
\Omega\left(s^{k(s)}\right) E(s, \rho)\left[s^{k(s) e} \rho\right]^{m+1} s^{-k(s)} \cdot s^{k(s) R} \cdot \exp [Q(s)]
$$

where $E(s, \rho)$ is bounded in (5.5) or (5.7) respectively, and $e$ is given in (3.44). 
For the last term $R_{m} v^{(m)}$ we have from (3.13) (5.8) and boundedness of the function $\hat{v}^{(m)}(s, \rho)$,

$$
R_{m} v^{(m)}=\Omega\left(s^{k(s)}\right) E(s, \rho)\left[s^{k(s) e} \rho\right]^{m+1} s^{-k(s)} s^{k(s) R} \exp [Q(s)],
$$

where $E(s, \rho)$ is bounded. Hence we have

$$
H_{m} v^{(m)}-v^{(m)^{\prime}}=\Omega\left(s^{k(s)}\right) E(s, \rho)\left[s^{k(s) e} \rho\right]^{m+1} s^{-k(s)} s^{k(s) R} \exp [Q(s)]
$$

with another bounded function $E(s, \rho)$. written

Using above results and (3.13) with $m=0$, the integral equation (5.13) can be

$$
\begin{aligned}
\hat{z}^{(m)}(s, \rho)=w_{0}(s) & \int_{\Gamma^{\prime}(s)} s^{k(s) R}\{\exp [Q(s)-Q(\sigma)]\} \sigma^{-k(\sigma) R} w_{0}(\sigma)^{-1}\left\{\sigma^{k(\sigma)(q / n+1 / n h)} \rho\right. \\
& \left.\cdot E_{1}(\sigma, \rho) \hat{z}^{(m)}(\sigma, \rho)+E(\sigma, \rho) \sigma^{k(\sigma) e(m+1)} \rho^{m+1} \sigma^{-k(\sigma)}\right\} \\
& \cdot \sigma^{k(\sigma) R}\{\exp [Q(\sigma)-Q(s)]\} s^{-k(s) R} d \sigma,
\end{aligned}
$$

where $w_{0}(\sigma)^{-1}, E_{1}(\sigma, \rho)$ and $E(\sigma, \rho)$ are bounded in the domain considered. We prove the existence of the solutions of this equation by the fixed point theorem. Let $F$ be the set of all matrices functions with the properties that every matrix function $Z=\left(z_{i j}(s, \rho)\right)$ is defined in $D_{2}$ except for $|s|=s_{0}$, holomorphic for $s \neq \infty$, and satisfies the inequalities in the domain (5.1) such that

$$
\begin{array}{lll}
\|\hat{Z}\| \equiv \max _{1 \leqq i \leqq n} \sum_{j=1}^{n}\left|z_{2 j}(s, \rho)\right| \leqq M|\rho|^{m+1}, & \text { if } & |s| \leqq s_{0}, \\
\|\hat{Z}\| \leqq M\left|s^{e} \rho\right|^{m+1}, & \text { if } & |s|>s_{0} .
\end{array}
$$

The constant $M$ and the other constants in (5.1) are defined in later.

The mapping $T$ is defined by

$$
\begin{aligned}
T(\hat{Z})=w_{0}(s) & \int_{\Gamma(s)} s^{k(s) R}\{\exp [Q(s)-Q(\sigma)]\} \sigma^{-k(\sigma) R} w_{0}(\sigma)^{-1}\left\{E_{1}(\sigma, \rho) \hat{Z}(\sigma, \rho) \sigma^{k(\sigma)(e-1)}\right. \\
& \left.+E(\sigma, \rho) \sigma^{k(\sigma) e(m+1)} \rho^{m+1} \sigma^{-k(\sigma)}\right\} \sigma^{k(\sigma) R}\{\exp [Q(\sigma)-Q(s)]\} s^{-k(s) R} d \sigma .
\end{aligned}
$$

We can write this equation for each component of $\hat{Z}=\left(\hat{z}_{2 j}(s, \rho)\right)$ as follows

$$
\begin{aligned}
T\left(\hat{z}_{2 j}\right)=s^{k(s)\left(r_{i}-r_{j}\right)} \int_{r_{i j(s)}} \sigma^{-k(\sigma)\left(r_{i}-r_{j}\right)}\left\{\exp \left[q_{i j}(s)-q_{i j}(\sigma)\right]\right\} \\
\cdot\left\{L_{2 j}(\hat{Z}) \sigma^{k(\sigma)(e-1)} \rho+p_{i j}(\sigma, \rho) \sigma^{k(\sigma) e(m+1)} \rho^{m+1} \sigma^{-k(\sigma)}\right\} d \sigma,
\end{aligned}
$$

where $r_{\imath}$ and $q_{i j}(s)$ are defined in (3.23) and (3.36). Here $L_{\imath j}(\hat{Z})$ is a linear form of $j$-th column of $\hat{Z}$ with bounded coefficients and $p_{i j}(\sigma, \rho)$ is a bounded matrix function.

If we transform $\sigma$ and $s$ into $\zeta$ and $\xi$ by the relations (3.38), the sector $S$ corresponds to the half plane $\Sigma$, and the integral (5.23) becomes

$$
\begin{aligned}
& T\left(\hat{z}_{\imath j}\right)=\xi^{k(s)\left(\pi_{i}-\pi_{\eta}\right)} \int_{\lambda_{i j(\xi)}} \zeta^{-k(\sigma)\left(\pi_{i}-\pi_{j}\right)}\left\{\exp \left(\lambda_{i}-\lambda_{j}\right)(\xi-\zeta)\right\} \\
& \quad \cdot\left\{\hat{L}_{\imath j}(\hat{Z}) \zeta^{k(\sigma)(e-1) n /(n+q)}+\hat{p}_{i j}(\sigma, \rho) \zeta^{k(\sigma)[e(m+1) n /(n+q)-n / n \vdash q]} \rho^{m+1}\right\} \zeta^{-q /(n+q)} d \zeta,
\end{aligned}
$$


where $\pi_{\imath}$ and $\lambda_{\imath}$ are defined at a) and b) in Section 3 , and $L_{\imath j}(\hat{Z})$ is a linear combination of $j$-th column of the matrix $Z$ with bounded coefficients and $\hat{p}_{i j}(\sigma, \rho)$ is a bounded matrix function.

Let $\mathscr{K}$ be a closed half disk in $\Sigma$ which satisfies

$$
\left|\zeta^{q e /(n+q)} \rho\right| \leqq \eta_{2}
$$

for each $\rho$, and $\eta_{2}$ denotes a constant sufficiently small. On the circular arc of the boundary of $\mathcal{K}$, we can conclude from the choice of $\alpha$ which determines the sector $\Sigma$ in (3.21) that there exists for every $i, j(i \neq j)$ a unique point $\zeta_{2 \jmath}$ at which $\operatorname{Re}\left[\left(\lambda_{i}-\lambda_{j}\right) \zeta\right]$ attains its maximum in $\mathscr{H}$. Then the quantity $\operatorname{Re}\left[\left(\lambda_{i}-\lambda_{j}\right)(\xi-\zeta)\right]$ increases monotonically when $\zeta$ moves from $\zeta_{2 j}$ to $\xi$ in $\mathscr{H}$ along a straight segment. Here we limit $\xi$ to the convex polygon $\mathscr{H}^{*}$ in $\mathscr{H}$ whose vertices are $\zeta_{\imath \jmath}$ and two endpoints of the diameter of $\mathscr{H}$. If $\xi$ is any point of $\mathscr{H} *$, the integral path $\lambda_{\imath j}(\xi)$ in (5.24) is defined by the segment joining $\xi$ and $\zeta_{2 \jmath}$. Thus for $\zeta$ on $\lambda_{\imath j}(\xi)$, there exist a positive constant $p$, independent of $i, j$ and $\rho$ such that

$$
\operatorname{Re}\left[\left(\lambda_{i}-\lambda_{j}\right)(\xi-\zeta)\right]<-p|\xi-\zeta| \text {. }
$$

We take here the inverse image of $\lambda_{i j}(\xi)$ in the $\sigma$-plane as the integral path $\gamma_{i j}(s)$ for $i \neq j$, and the path $\gamma_{j j}(s)$ is to be the ray from the origin to $s$.

Here we choose the positive constants $\rho_{2}$ and $\delta_{2}$ in (5.1) so small that the domain defined by

$$
0<|\rho| \leqq \rho_{2}, \quad|\arg \rho| \leqq \delta_{2}
$$

satisfies the condition (1.3).

Now we prove the contracting property of the transformation $T$. From (5.24) and (5.20), or (5.21),

$$
\begin{aligned}
\left|T\left(\hat{z}_{i j}\right)\right| \leqq|\rho|^{m+1}\left|\xi^{k(s)\left(\pi_{i}-\pi_{j}\right)}\right| & \int_{\lambda_{i j}(\xi)}\left|\zeta^{-k(\sigma)\left(\pi_{i}-\pi_{j}\right)}\right|\left\{\exp \operatorname{Re}\left(\lambda_{i}-\lambda_{j}\right)(\xi-\zeta)\right\} \\
& \cdot\left\{M|\zeta|^{k(\sigma) e n /(n+q)}|\rho|+B\right\}|\zeta|^{k(\sigma)[e(m+1) n /(n ! q)-n /(n+q)]}|\zeta|^{-q /(n+q)}|d \zeta|,
\end{aligned}
$$

where $B$ is some constant. We estimate the the integral:

$$
J \equiv \int_{\lambda_{i j(\xi)}}\left|\zeta^{-k(\sigma)\left(\pi_{i}-\pi_{j}\right)}\right|\left\{\exp \operatorname{Re}\left(\lambda_{i}-\lambda_{j}\right)(\xi-\zeta)\right\}|\zeta|^{k(\sigma) h(m)}|\zeta|^{-q /(n+q)}|d \zeta|,
$$

where $h(m)=e(m+1) n /(n+q)-n /(n+q)$.

(1) $j \neq k$. From $(5.26)$ we can write for each $\xi \in \mathcal{H}^{*}$

$$
J \leqq C_{1} \int_{\lambda_{i j(\xi)}}\{\exp -p|\xi-\zeta|\}|\zeta|^{-k(o)\left\{\operatorname{Re}\left(\pi_{i}-\pi j\right)-h(m)\right\}}|\zeta|^{-q /(n+q)}|d \zeta|
$$

where $C_{1}$ and other constants $C_{r}$ introduced below are some constants independent of $\xi$ and $\rho$. If the point $\xi$, and all the points of $\lambda_{2 j}(\xi)$ satisfy the inequality

we can prove easily that

$$
|\xi|>\xi_{0}=\frac{n}{n+q} s_{0}^{(n+q) / n},
$$

$$
\begin{aligned}
J & \leqq C_{2}|\xi|^{-\operatorname{Re}\left(\pi_{i}-\pi_{j}\right)+e(m \mid 1) n /(n \mid q)-1} \\
& \leqq C_{2}|\xi|^{-\operatorname{Re}\left(\pi_{i}-\pi_{j}\right) \mid(m+1) \rho n /(n+q)},
\end{aligned}
$$


and if $|\xi|>\xi_{0}$, and some parts of $\lambda_{\imath j}(\xi)$ are contained in $|\xi| \leqq \xi_{0}$,

$$
J \leqq C_{3} \xi_{0}{ }^{n /(n+q)} .
$$

Next, if $|\xi| \leqq \xi_{0}$ and $\lambda_{\imath j}(\xi)$ is contained in $|\xi| \leqq \xi_{0}$,

$$
J \leqq C_{4} \xi_{0} n /(n+q),
$$

and if $|\xi| \leqq \xi_{0}$, and $\lambda_{\imath j}(\xi)$ has the parts on which $|\xi|>\xi_{0}$,

$$
J \leqq C_{5} \xi_{0}-\operatorname{Re}\left(\pi_{i}-\pi_{i}\right)+(m+1) e n /(n+q) .
$$

(2) $i=j$. In this case $J$ becomes

If $|\xi| \leqq \xi_{0}$, we have

$$
J=\int_{\lambda_{i i(\xi)}}|\zeta|^{k(\sigma) h(m)-q /(n+q)}|d \zeta|
$$

$$
J \leqq C_{6} \xi_{0} n /(n+q) .
$$

If $|\xi|>\xi_{0}$,

$$
J \leqq C_{7}|\xi|^{(m+1) \rho n /(n+q)} .
$$

Then for each case, it follows that

$$
J \leqq C_{8}|\xi|^{k(s)(m+1) e n /(n+q)}|\xi|^{-k(s) \operatorname{Re}\left(\pi_{i}-\pi j\right)},
$$

where $C_{8}$ is some constant depending on $\xi_{0}$ and $m$, but is independent of $\rho$ and $\xi$. Therefore we have from $(5.28)$ that

$$
\left|T\left(\hat{z}_{2 j}\right)\right| \leqq C_{0}\left\{M\left|s^{k(s) e} \rho\right|+B\right\}\left|s^{k(s) e} \rho\right|^{m \vdash 1},
$$

and then,

$$
\|T(\hat{Z})\| \leqq n C_{0}\left\{M\left|s^{k(s) e} \rho\right|+B\right\}\left|s^{k(s) e} \rho\right|^{m+1} .
$$

If we choose $M$ so large and $\eta_{1}, \rho_{2}$ so small that

and

$$
n C_{0}\left\{M \eta_{1}+B\right\} \leqq M
$$

$$
n C_{0}\left\{M \delta_{2}+B\right\} \leqq M,
$$

so we get the contracting property, $\|T(F)\| \leqq M\left|s^{k(s) e} \rho\right|^{m+1}$. The fact that $T(Z)$ is holomorphic matrix function in the domain $D_{2}$ except for $|s|=s_{0}$ and $s=\infty$ follows from the method of construction of the mapping $T$, and from the uniform convergence of the integral (5.22).

Hence we obtain a solution of the integral equation (5.13) and then a solution of (5.11) which is also a solution of the differential equation (5.10).

If we put

$$
v(s, \rho)=v^{(m)}(s, \rho)+z^{(m)}(s, \rho),
$$

then $v(s, \rho)$ is a solution of the differential equation (3. 8). The facts that the solution $z^{(m)}(s, \rho)$ of $(5.10)$ in $F$ is unique and then the solution $v(s, \rho)$ of $(3.8)$ is independent of $m$, are easily established as in Section 4 .

The constant $c_{3}$ in (5.1) is chosen such that $c_{3} \leqq \eta_{1}$ and the inverse image $H^{*}$ in the $s, \rho$-plane of $\mathcal{H}^{*}$ in the $\xi, \rho$-plane contains the domain (5.1). 


\section{$\S 6$. Conclusions.}

The differential equation (1.1) has two types of asymptotic solutions. The one is from Theorem 4. 1 ,

$$
\begin{aligned}
& y=\Omega(x) \cdot u \\
& u=\hat{u}(\tau, \varepsilon) \exp \left[\sum_{\nu=0}^{h} \varepsilon^{\nu-h} F_{\nu}(\tau)\right] \\
& \hat{u}(\tau, \varepsilon) \simeq \sum_{\nu=0}^{\infty} u_{\nu}(\tau) \varepsilon^{\nu},
\end{aligned}
$$

where $x=\tau^{a b}$, and $u_{\nu}(\tau)$ are defined in Theorem (2. 1). The domain of the asymptotic expansion is given (4.2). And the other is from Theorem 5.1,

$$
\begin{aligned}
& y=\Omega\left(\varepsilon^{a}\right) v(s, \rho) \\
& v(s, \rho) \simeq \Omega\left(s^{k(s)}\right) \sum_{\nu=0}^{\infty} w_{\nu}(s)\left[s^{k(s) e} \rho\right]^{\nu} s^{k((s) R} \exp [Q(s)],
\end{aligned}
$$

where $x=\varepsilon^{a} s$ and $\varepsilon=\rho^{n+q}$. The domain of the asymptotic expansion is given in (5.1). Now we prove that the two domains (4.2) and (5.1) overlap with each other for all sufficiently small $\varepsilon$.

The inequality $c_{1}|\varepsilon|^{1 / b} \leqq|\tau| \leqq c_{2}$ becomes in terms of $x$ and $\varepsilon$

$$
c_{1}^{a b}|\varepsilon|_{a} \leqq|x| \leqq c_{2}{ }^{a b},
$$

and the inequality $\left|s^{e} \rho\right| \leqq c_{3}$ becomes

$$
|x|=c_{3}^{1 / e}|\varepsilon|^{a-1 / e(n+q)},
$$

where $e=1 / n h+q / n+1>0$. The domains (6.6) and (6.7) overlap clearly for all $\varepsilon$ sufficiently small, and this is the chief purpose of this paper.

Now we have in mind that the result of this paper is applicable th a boundary value problem of a linear differential equation containing a turning point, and that this matching method may be developed to a certain singular perturbation problem of linear and nonlinear systems which contains the turning point problem as a special case. In future, we will discuss these problems.

\section{REFERENCES}

[1] Iwano M., And Y. Sibuya, Reduction of the order of a linear ordinary differential equations containing a parameter. Kōdai Math. Sem. Rep. 15 (1963), 1-28.

[2] Nishimoto, T., On matching methods in turning point problems. Kōdai Math. Sem. Rep. 17 (1965), 198-221.

[3] Окиво, K., A global representation of a fundamental set of solutions and a Stokes phenomenon for a system of linear ordinary differential equations. J. Math. Soc. Japan 15 (3) (1963), 268-288.

[4] Wasow, W., Turning point problems for system of linear differential equations. Part 1: The formal theory. Comm. Pure and App. Math. 14 (1961), 657-673.

[5] Wasow, W., Turning point problems for systems of linear differential equations. Part II: The analytic theory. Comm. Pure and App. Math. 15 (1962), 173-187.

Department of Mathematics, Tokyo Institute of Technology. 